\title{
The McDonald Gompertz Distribution: Properties and
}

\author{
Applications \\ Rasool Roozegar, Saeid Tahmasebi ${ }^{\dagger}$, and Ali Akbar Jafari* \\ * Department of Statistics, Yazd University, Yazd, Iran \\ ${ }^{\dagger}$ Department of Statistics, Persian Gulf University, Bushehr, Iran
}

\begin{abstract}
This paper introduces a five-parameter lifetime model with increasing, decreasing, upside -down bathtub and bathtub shaped failure rate called as the McDonald Gompertz (McG) distribution. This new distribution extend the Gompertz, generalized Gompertz, generalized exponential, beta Gompertz and Kumaraswamy Gompertz distributions, among several other models. We obtain several properties of the McG distribution including moments, entropies, quantile and generating functions. We provide the density function of the order statistics and their moments. The parameter estimation is based on the usual maximum likelihood approach. We also provide the observed information matrix and discuss inferences issues. In the end, the flexibility and usefulness of the new distribution is illustrated by means of application to two real data sets.
\end{abstract}

Keywords: Gompertz distribution; McDonald distribution; Maximum likelihood estimation; Kumaraswamy distribution; Moment generating function; Entropy.

2010 AMS Subject Classification: 62E15, 60E05, 62F10.

\section{Introduction}

The Gompertz $(\mathrm{G})$ distribution, generalizing exponential (E) distribution, is a popular distribution that has been commonly used in many applied problems for modeling data in biology Economos (1982), gerontology Brown and Forbes (1974), engineering and marketing studies Bemmaor and Glady (2012). A significant progress has been made towards the generalization and construction flexible distributions to facilitate better modeling of well-known lifetime data. The book by Johnson et al. (1995) provides some applications of the G distribution.

${ }^{*}$ Corresponding: rroozegar@yazd.ac.ir 
In recent years, many authors have proposed distributions which can arise as special submodels within the McDonald generated or generalized beta generated (GBG) class of distributions. Alexander et al. (2012) introduce a class of generalized beta-generated distributions that have three shape parameters in the generator. They considered eleven different parents: normal, log-normal, skewed student-t, Laplace, exponential, Weibull, Gumbel, Birnbaum-Saunders, gamma, Pareto and logistic distributions. Other generalizations are McDonald gamma distribution by Marciano et al. (2012), McDonald inverted beta distribution by Cordeiro and Lemonte (2012), McDonald normal distribution by Cordeiro et al. (2012a), McDonald extended exponential distribution by Cordeiro et al. (2012b), McDonald half-logistic distribution by Oliveira et al. (2013), McDonald Dagum by Oluvede and Rajasooriva (2013), McDonald generalized beta-binomial distribution by Manoj et al. (2013), McDonald log-logistic distribution by Tahir et al. (2014), McDonald arcsine distribution by Cordeiro and Lemonte (2014), and McDonald Weibull distribution by Cordeiro et al. (2014). One of the advantages of the McDonald generated distribution lies in its ability of fitting skewed data such as other wellknown distributions in the literature Mudholkar and Natarajan (2002); Mudholkar and Wang (2007).

In this paper, we introduce a new five-parameter model called the McDonald Gompertz (McG) distribution that includes as special sub-models some recent distributions in the literature. This distribution offers a more flexible distribution for modeling lifetime data in terms of its hazard rate shapes that are decreasing, increasing, upside-down bathtub and bathtub shaped. Several mathematical properties of this new model in order to attract wider applications in reliability, engineering and in other areas of research are provided.

This paper is organized as follows. In Section 2, we introduce the McG distribution, density and hazard functions. Some special models of the new distribution are described in this section. In Section 3, we present useful expansions and properties of the cumulative distribution function (cdf), probability density function (pdf), $k$ th moment and moment generating function of the McG distribution. Moreover, order statistics and their moments, entropy and quantile measures are provided in this section. Estimation of the McG parameters by maximum likelihood (ML) method is described in Section 4. Finally, application of the McG model using two real data sets are considered in Section 5 , 


\section{The McG model}

The generalized beta distribution of the first kind (or beta type I) or McDonald distribution was introduced by McDonald (1984). The cdf of the McDonald distribution is given by

$$
F(x)=I\left(x^{c} ; a / c, b\right), \quad 0<x<1, \quad a, b, c>0
$$

where $I(y ; a, b)=\frac{B_{y}(a, b)}{B(a, b)}=\frac{1}{B(a, b)} \int_{0}^{y} w^{a-1}(1-w)^{b-1} d w$ is the incomplete beta function ratio of type $\mathrm{I}$ and $B(a, b)=\int_{0}^{1} w^{a-1}(1-w)^{b-1} d w$ is the beta function.

The cdf of McG model can be defined by

$$
F(y ; a, b, c, \theta, \gamma)=I\left(\left[1-\exp \left(-\frac{\theta}{\gamma}\left(e^{\gamma y}-1\right)\right)\right]^{c} ; a / c, b\right), \quad y>0,
$$

where $\theta, \gamma>0$. The pdf corresponding to (2.1) is given by

$$
\begin{aligned}
f(y ; a, b, c, \theta, \gamma)= & \frac{c \theta e^{\gamma y}}{B(a / c, b)} \exp \left(-\frac{\theta}{\gamma}\left(e^{\gamma y}-1\right)\right)\left[1-\exp \left(-\frac{\theta}{\gamma}\left(e^{\gamma y}-1\right)\right)\right]^{a-1} \\
& \times\left\{1-\left[1-\exp \left(-\frac{\theta}{\gamma}\left(e^{\gamma y}-1\right)\right)\right]^{c}\right\}^{b-1} .
\end{aligned}
$$

Here after, we denote a random variable $Y$ with pdf in (2.2) by $M c G(a, b, c, \theta, \gamma)$. Indeed, the McG distribution belongs to McDonald-generalized class of distributions with cdf and pdf as

$$
F(y)=I\left(G^{c}(y) ; a / c, b\right)=\frac{1}{B(a / c, b)} \int_{0}^{G^{c}(y)} w^{a / c-1}(1-w)^{b-1} d w,
$$

and

$$
f(y)=\frac{c}{B(a / c, b)} g(y) G^{a-1}(y)\left(1-G^{c}(y)\right)^{b-1},
$$

respectively. The cdf in (2.1) can be expressed in terms of the hypergeometric function as

$$
F(y ; a, b, c, \theta, \gamma)=\frac{c G^{a}(y)}{a B(a / c, b)}{ }_{2} F_{1}\left(a / c, 1-b ; a / c+1, G^{c}(y)\right)
$$

where ${ }_{2} F_{1}(a, b ; c, x)=\sum_{n=0}^{\infty} \frac{(a)_{n}(b)_{n}}{(c)_{n}} \frac{x^{n}}{n !}$ is ascending factorial, and $G(y)=1-\exp \left(-\frac{\theta}{\gamma}\left(e^{\gamma y}-1\right)\right)$ is the cdf of $\mathrm{G}$ distribution.

Theorem 2.1. Let $f(y ; a, b, c, \theta, \gamma)$ be the pdf of $M c G$ distribution given by (2.2). The limiting behavior of $f(y ; a, b, c, \theta, \gamma)$ for different values of its parameters is given below:

$i$. If $a=1$, then $\lim _{y \rightarrow 0^{+}} f(y ; a, b, c, \theta, \gamma)=\frac{\theta c}{B(1 / c, b)}$.

ii. If $a>1$, then $\lim _{y \rightarrow 0^{+}} f(y ; a, b, c, \theta, \gamma)=0$.

iii. If $a<1$, then $\lim _{y \rightarrow 0^{+}} f(y ; a, b, c, \theta, \gamma)=\infty$.

$i v . \lim _{y \rightarrow+\infty} f(y ; a, b, c, \theta, \gamma)=0$. 
Proof. The parts (i)-(iii) are obviously proved. For part (iv), we have

$$
\begin{aligned}
& 0 \leq\left[1-\left[1-\exp \left\{-\frac{\theta}{\gamma}\left(e^{\gamma y}-1\right)\right\}\right]^{c}\right]^{b-1}<1 \Longrightarrow \\
& 0<f(y ; a, b, c, \theta, \gamma)<c \theta e^{\gamma y} \exp \left(-\frac{\theta}{\gamma}\left(e^{\gamma y}-1\right)\right)\left[1-\exp \left(-\frac{\theta}{\gamma}\left(e^{\gamma y}-1\right)\right)\right]^{a-1} / B(a / c, b) .
\end{aligned}
$$

It can be easily shown that

$$
\lim _{y \rightarrow \infty} c \theta e^{\gamma y} \exp \left(-\frac{\theta}{\gamma}\left(e^{\gamma y}-1\right)\right)\left[1-\exp \left(-\frac{\theta}{\gamma}\left(e^{\gamma y}-1\right)\right)\right]^{a-1}=0,
$$

and the proof is completed.
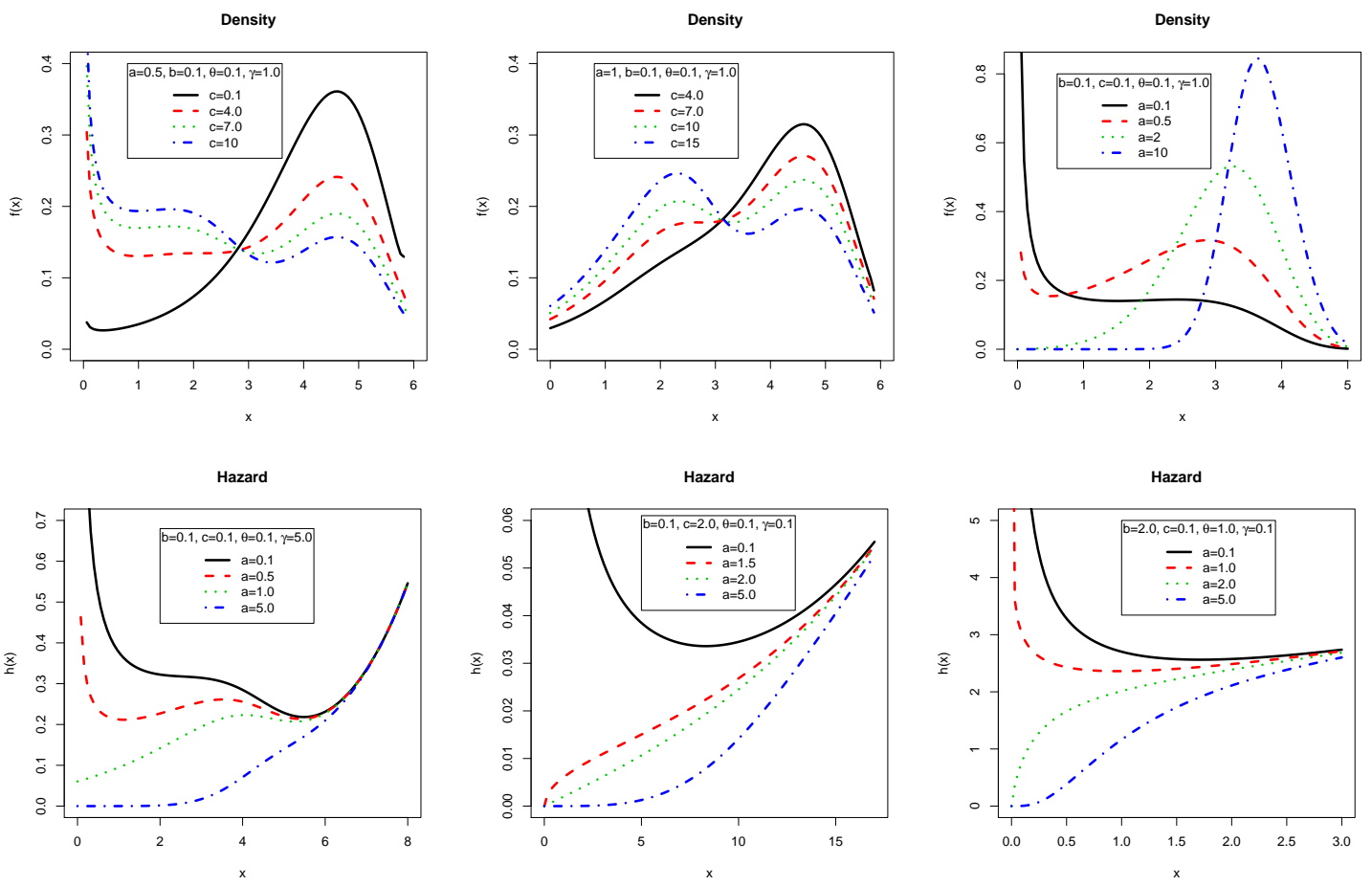

Figure 1: pdf and hrf of McG model for some values of parameters.

From equations (2.1) and (2.2), it is easy to verify that the hazard rate function (hrf) of the McG distribution is given by

$$
\begin{aligned}
h(y ; a, b, c, \theta, \gamma)= & \frac{c \theta e^{\gamma y} \exp \left(-\frac{\theta}{\gamma}\left(e^{\gamma y}-1\right)\right)}{B(a / c, b)-B_{\left[1-\exp \left(-\frac{\theta}{\gamma}\left(e^{\gamma y}-1\right)\right)\right]^{c}}(a / c, b)} \\
& \times\left[1-\exp \left(-\frac{\theta}{\gamma}\left(e^{\gamma y}-1\right)\right)\right]^{a-1}\left[1-\left(1-\exp \left(-\frac{\theta}{\gamma}\left(e^{\gamma y}-1\right)\right)\right)^{c}\right]^{b-1}, \quad y>0,
\end{aligned}
$$

and the corresponding reversed hazard rate function reduces to

$$
r(y ; a, b, c, \theta, \gamma)=\frac{c \theta e^{\gamma y} \exp \left(-\frac{\theta}{\gamma}\left(e^{\gamma y}-1\right)\right)}{B_{\left[1-\exp \left(-\frac{\theta}{\gamma}\left(e^{\gamma y}-1\right)\right)\right]^{c}}(a / c, b)}\left[1-\exp \left(-\frac{\theta}{\gamma}\left(e^{\gamma y}-1\right)\right)\right]^{a-1}
$$




$$
\times\left[1-\left(1-\exp \left(-\frac{\theta}{\gamma}\left(e^{\gamma y}-1\right)\right)\right)^{c}\right]^{b-1}, \quad y>0 .
$$

Figure[1illustrates some of the possible shapes of density and hazard functions for selected values of parameters. For instance, these plots show the hazard function of the new model is much more flexible than the beta Gompertz (BG) and G distributions. The hazard rate function can be bathtub shaped, monotonically increasing or decreasing and upside-down bathtub shaped depending on the parameter values.

The McG distribution contains as sub-models the Kumaraswamy Gompertz (KumG), the BG Jafari et al. (2014), and the beta generalized exponential (BGE) or McE Barreto-Souza et al. (2010) distributions for $c=1, a=c$ and $\gamma \rightarrow 0$, respectively. It also contains the beta exponential (BE) Nadarajah and Kotz (2006), the generalized Gompertz (GG) El-Gohary et al. (2013), and the Kumaraswamy exponential (KumE) Nadarajah et al. (2012) distributions. The GE Gupta and Kundu (1999), the G and E distributions are also sub-models. The classes of distributions that are included as special sub-models of the McG distribution are displayed in Figure 2 ,

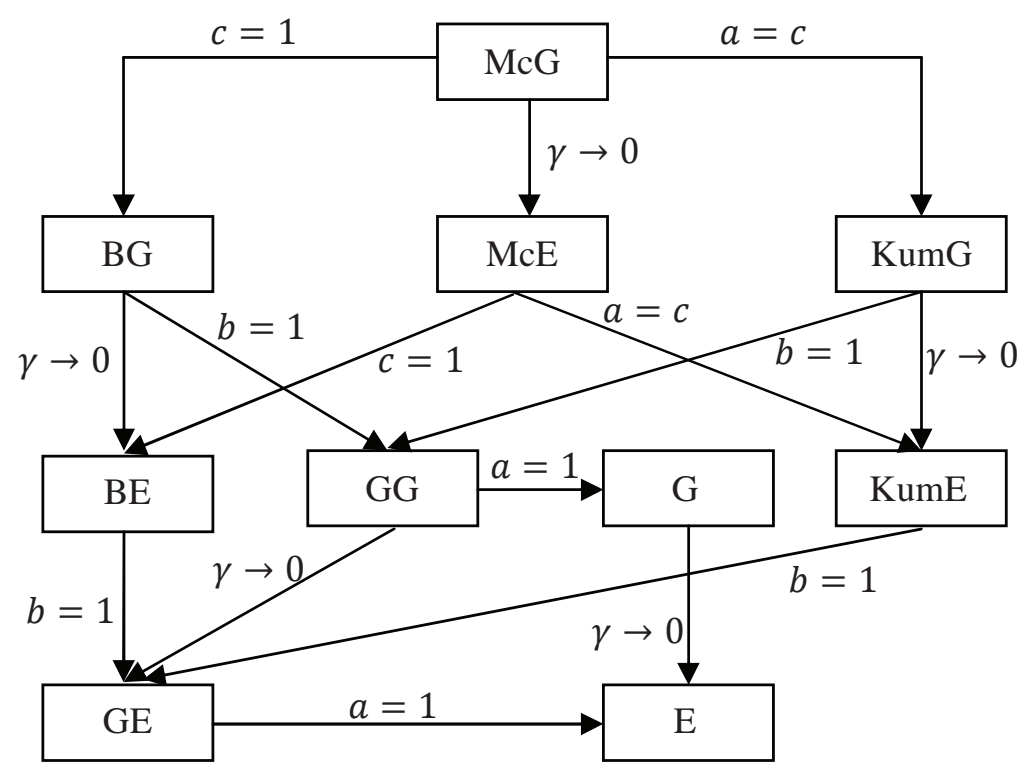

Figure 2: Relationships of the McG sub-models.

If the random variable $Y$ has the McG distribution, then it has the following properties:

1. The random variable $V=\left[1-\exp \left(-\frac{\theta}{\gamma}\left(e^{\gamma Y}-1\right)\right)\right]^{c}$ satisfies the beta distribution with parameters $a / c$ and $b$. Therefore, the random variable $T=\frac{\theta}{\gamma}\left(e^{\gamma Y}-1\right)$ has the BGE (or McG) distribution Barreto-Souza et al. (2010). Furthermore, the random variable $Y=G^{-1}\left(V^{1 / c}\right)=$ 
$\frac{1}{\gamma} \log \left[1-\frac{\gamma}{\theta} \log \left(1-V^{1 / c}\right)\right]$ follows McG distribution. This result helps us in simulating data from McG distribution. The plots comparing the exact McG density function and the histogram from a simulated data set with size 100 for some parameter values are given in Figure 3 (left). Also, the plots of empirical distribution function and exact distribution function are given in Figure 3 (right). These plots indicate that the simulated values are consistent with the McG distribution.

2. If $a=i$ and $b=n-i+1$, where $i$ and $n$ are positive integer values, then the $F(y ; a, b, c, \theta, \gamma)$ is the cdf of the $i$ th order statistic of GG distribution.
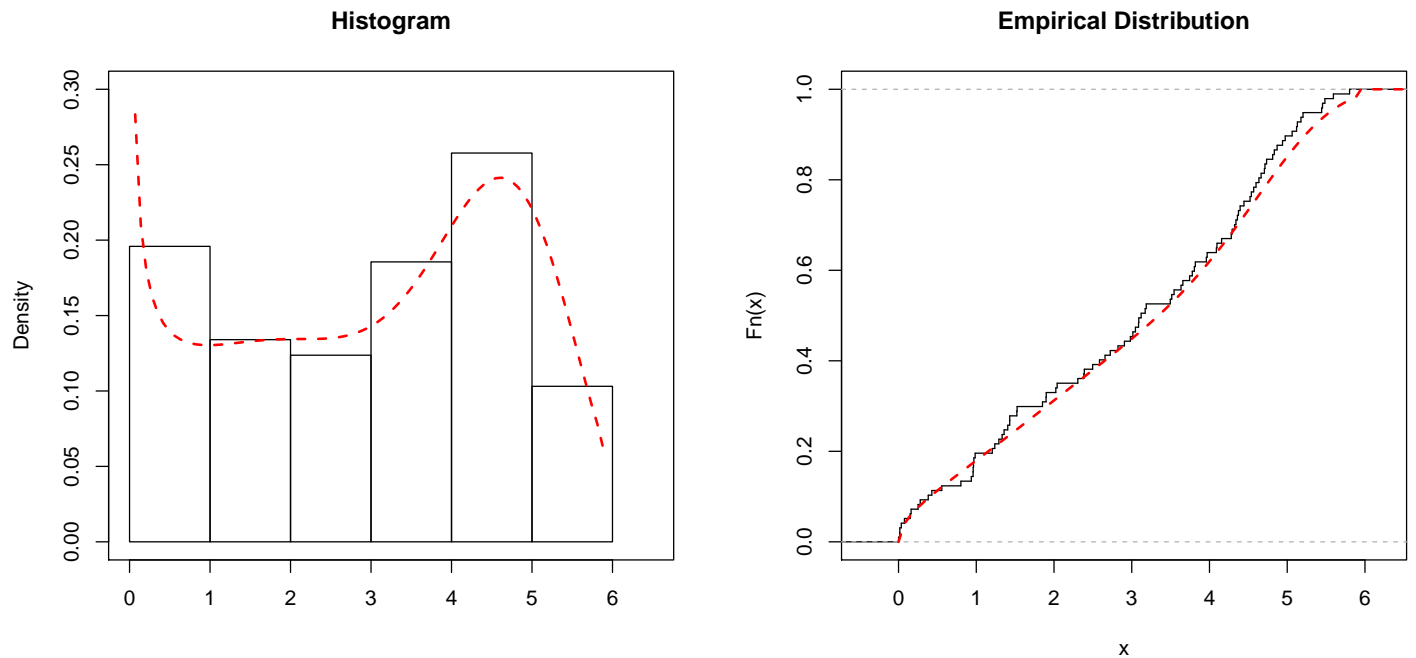

Figure 3: The histogram of a generated data set with size 100 and the exact McG density (left) and the empirical distribution function and exact distribution function (right).

\section{General properties}

In this section, some properties of McG distribution are considered.

\subsection{A useful expansion}

We derive some expansions for the cdf, $k$ th moment and moment generating function of the McG distribution. The binomial series expansion is defined by

$$
(1-z)^{m}=\sum_{j=0}^{\infty}(-1)^{j}\left(\begin{array}{c}
j \\
m
\end{array}\right) z^{j}=\sum_{j=0}^{\infty}(-1)^{j} \frac{\Gamma(m+1)}{\Gamma(m-j+1)} \frac{z^{j}}{j !},
$$

where $|z|<1$ and $m$ is a positive real non-integer.

The following proposition reveals that the $\mathrm{McG}$ distribution can be expressed as a mixture of distribution function of GG distribution, whereas Proposition 3.2 provides a useful expansion 
for the pdf in (2.2).

Proposition 3.1. The cdf in (2.1) is a mixture of distribution function of $G G$ distribution on the form

$$
F(y ; a, b, c, \theta, \gamma)=\sum_{j=0}^{\infty} p_{j}[G(y)]^{a+j c}=\sum_{j=0}^{\infty} p_{j} G_{j}(y),
$$

where $p_{j}=\frac{(-1)^{j} \Gamma(b)}{B(a / c, b) \Gamma(b-j) j !(a / c+j)}$ and $G_{j}(y)=(G(y))^{a+j c}$ is the distribution function of a random variable which has a $G G$ distribution with parameters $\theta, \gamma$ and $a+j c$.

using binomial expansion (3.1) to the term $[G(y)]^{a+j c}$ in (3.2), we have

$$
\begin{aligned}
{[G(y)]^{a+j c} } & =\sum_{k=0}^{\infty}(-1)^{k}\left(\begin{array}{c}
a+j c \\
k
\end{array}\right)(1-G(y))^{k} \\
& =\sum_{r=0}^{\infty} \sum_{k=r}^{\infty}(-1)^{k}\left(\begin{array}{c}
a+j c \\
k
\end{array}\right)\left(\begin{array}{l}
k \\
r
\end{array}\right)[G(y)]^{r} .
\end{aligned}
$$

Now, (3.2) becomes

$$
F(y ; a, b, c, \theta, \gamma)=\sum_{j=0}^{\infty} \sum_{r=0}^{\infty} \sum_{k=r}^{\infty} p_{j}(-1)^{k+r}\left(\begin{array}{c}
a+j c \\
k
\end{array}\right)\left(\begin{array}{l}
k \\
r
\end{array}\right)[G(y)]^{r}=\sum_{r=0}^{\infty} b_{r}[G(y)]^{r},
$$

where $b_{r}=\sum_{j=0}^{\infty} \sum_{k=r}^{\infty} p_{j}(-1)^{k+r}\left(\begin{array}{c}a+j c \\ k\end{array}\right)\left(\begin{array}{l}k \\ r\end{array}\right)$.

Proposition 3.2. The pdf of $M c G$ can be expressed as an infinite mixture of $G G$ densities with parameters $\theta, \gamma$ and $(a+j c)$ given by

$$
f(y ; a, b, c, \theta, \gamma)=\sum_{j=0}^{\infty} p_{j}(a+j c) g(y)[G(y)]^{a+j c-1}=\sum_{r=0}^{\infty} p_{j} g_{j}(y),
$$

where $g_{j}(y)=(a+j c) g(y)[G(y)]^{a+j c-1}$. We can write the pdf of $M c G$ as

$$
f(y ; a, b, c, \theta, \gamma)=g(y) \sum_{r=0}^{\infty} c_{r}[G(y)]^{r},
$$

where $c_{r}=\sum_{j=0}^{\infty} \sum_{k=r}^{\infty}(-1)^{j+k+r} \frac{c \Gamma(b)}{B(a / c, b) j ! \Gamma(b-j)}\left(\begin{array}{c}a+j c-1 \\ k\end{array}\right)\left(\begin{array}{l}k \\ r\end{array}\right)$.

\subsection{Moments and generating function}

In this section, we deal with the basic statistical properties of McG distribution such as the $k$-th moment and generating function in the following propositions.

Proposition 3.3. The $k$-th moment of $M c G$ distribution can be expressed as a infinite mixture of the $k$-th moment of $G G$ distributions as follows:

$$
E\left(Y^{k}\right)=\int_{0}^{\infty} y^{k} \sum_{j=0}^{\infty} p_{j}(a+j c) g(y)[G(y)]^{a+j c-1}=\sum_{j=0}^{\infty} p_{j} E\left(Y_{j}^{k}\right)
$$


where

$$
E\left(Y_{j}^{k}\right)=u_{j k} \sum_{i=0}^{\infty} \sum_{r=0}^{\infty}\left(\begin{array}{c}
a+j c-1 \\
i
\end{array}\right) \frac{(-1)^{i+r}}{\Gamma(r+1)} e^{\frac{\theta}{\gamma}(i+1)}\left[\frac{\theta}{\gamma}(i+1)\right]^{r}\left[\frac{-1}{\gamma(r+1)}\right]^{k+1},
$$

and $u_{j k}=\theta(a+j c) \Gamma(k+1)$.

Proposition 3.4. An explicit expression for the moment generating function of McG distribution follows from Proposition 3.2,

$$
M_{Y}(t)=\int_{0}^{\infty} e^{t x} \sum_{j=0}^{\infty} p_{j}(a+j c) g(y)[G(y)]^{a+j c-1}=\sum_{j=0}^{\infty} p_{j} M_{Y_{j}}(t),
$$

where

$$
M_{Y_{j}}(t)=\frac{(a+j c) \theta}{\gamma} \sum_{i=0}^{\infty} \sum_{k=0}^{\infty}(-1)^{i}\left(\begin{array}{c}
a+j c-1 \\
i
\end{array}\right)\left(\begin{array}{c}
t / \gamma \\
k
\end{array}\right) \frac{\Gamma(k+1)}{\left[\frac{(a+j c) \theta}{\gamma}\right]^{k+1}} .
$$

\subsection{Order statistics}

Order statistics make their appearance in many areas of statistical theory and practice. Let the random variable $Y_{i: n}$ be the $i$ th order statistic $\left(Y_{1: n} \leq Y_{2: n} \leq \cdots \leq Y_{n: n}\right)$ in a sample of size $n$ from the McG distribution. The pdf and cdf of $Y_{i: n}$ for $i=1,2, \ldots, n$ are given by

$$
\begin{aligned}
f_{i: n}(y) & =\frac{1}{B(i, n-i+1)} f(y)[F(y)]^{i-1}[1-F(y)]^{n-i} \\
& =\frac{1}{B(i, n-i+1)} \sum_{k=0}^{n-i}\left(\begin{array}{c}
n-i \\
k
\end{array}\right)(-1)^{k} f(y)[F(y)]^{k+i-1},
\end{aligned}
$$

and

$$
F_{i: n}(y)=\int_{0}^{y} f_{i: n}(t) d t=\frac{1}{B(i, n-i+1)} \sum_{k=0}^{n-i} \frac{(-1)^{k}}{k+i}\left(\begin{array}{c}
n-i \\
k
\end{array}\right)[F(y)]^{k+i},
$$

respectively, where $F(y)=\sum_{r=0}^{\infty} b_{r} G(y)$. We use throughout an equation by (Gradshteyn and Ryzhik (2007), page 17) for a power series raised to a positive integer $m$ given by

$$
\left(\sum_{r=0}^{\infty} b_{r} u^{r}\right)^{m}=\sum_{r=0}^{\infty} c_{m, r} u^{r}
$$

where the coefficients $c_{m, r}$ (for $r=1,2, \ldots$ ) are easily determined from the recurrence equation

$$
c_{m, r}=\left(r b_{0}\right)^{-1} \sum_{k=1}^{r}[k(m+1)-r+k] b_{k} c_{m, r-k}
$$

where $c_{m, 0}=b_{0}^{m}$. Hence, the coefficients $c_{m, r}$ can be calculated from $c_{m, 0}, \ldots, c_{m, r-1}$ and therefore, from the quantities $b_{0}, \ldots, b_{r}$. Using (3.5), the equations (3.3) and (3.4) can be written as

$$
f_{i: n}(y)=\frac{1}{B(i, n-i+1)} \sum_{k=0}^{n-i} \sum_{r=1}^{\infty} \frac{r}{k+i}(-1)^{k}\left(\begin{array}{c}
n-i \\
k
\end{array}\right) c_{i+k, r} g(y)[G(y)]^{r-1}
$$




$$
F_{i: n}(y)=\frac{1}{B(i, n-i+1)} \sum_{k=0}^{n-i} \sum_{r=0}^{\infty} \frac{1}{k+i}(-1)^{k}\left(\begin{array}{c}
n-i \\
k
\end{array}\right) c_{i+k, r}[G(y)]^{r} .
$$

An explicit expression for the $s$ th moments of $Y_{i: n}$ can be obtained as

$$
\begin{aligned}
E\left[Y_{i: n}^{s}\right]= & \frac{1}{B(i, n-i+1)} \sum_{k=0}^{n-i} \sum_{r=1}^{\infty} \frac{r}{k+i}(-1)^{k}\left(\begin{array}{c}
n-i \\
k
\end{array}\right) c_{i+k, r} \int_{0}^{+\infty} t^{s} g(t)[G(t)]^{r-1} d t \\
= & \frac{\theta \Gamma(s+1)}{B(i, n-i+1)} \sum_{k=0}^{n-i} \sum_{r=1}^{\infty} \frac{r}{k+i}(-1)^{k}\left(\begin{array}{c}
n-i \\
k
\end{array}\right) c_{i+k, r} \\
& \times \sum_{i_{1}=0}^{\infty} \sum_{i_{2}=0}^{\infty}\left(\begin{array}{c}
r \lambda-1 \\
i_{1}
\end{array}\right) \frac{(-1)^{i_{1}+i_{2}}}{\Gamma\left(i_{2}+1\right)} e^{\frac{\theta}{\gamma}\left(i_{1}+1\right)}\left[\frac{\theta\left(i_{1}+1\right)}{\gamma}\right]^{i_{2}}\left[\frac{-1}{\gamma\left(i_{2}+1\right)}\right]^{s+1} .
\end{aligned}
$$

\subsection{Quantile measures}

In this section, we consider the effect of each shape parameters $a, b$ and $c$ on the skewness and kurtosis of the McG distribution. To illustrate this effect, we use measures based on quantiles. The quantile function of the $M c G(a, b, c, \gamma, \theta)$ distribution say $Q(t)$ can be obtained as

$$
Q(t)=\frac{1}{\gamma} \log \left(1-\frac{\gamma}{\theta} \log \left(1-Q_{a / c, b}^{\frac{1}{c}}(t)\right)\right), \quad 0<t<1,
$$

where $Q_{a / c, b}(t)=I_{t}^{-1}(a / c, b)$ denotes the $t$ th quantile of beta distribution with parameters $a / c$ and $b$. The Bowley skewness (see Kenney and Keeping (1962)) based on quantiles can be calculated by

and the Moors kurtosis (see Moors (1988)) is defined as

$$
\mathcal{B}=\frac{Q\left(\frac{3}{4}\right)-2 Q\left(\frac{1}{2}\right)+Q\left(\frac{1}{4}\right)}{Q\left(\frac{3}{4}\right)-Q\left(\frac{1}{4}\right)},
$$

$$
\mathcal{M}=\frac{Q\left(\frac{7}{8}\right)-Q\left(\frac{5}{8}\right)+Q\left(\frac{3}{8}\right)-Q\left(\frac{1}{8}\right)}{Q\left(\frac{6}{8}\right)-Q\left(\frac{2}{8}\right)},
$$

where $Q($.$) denotes the quantile function. These measures are less sensitive to outliers and$ they exist even for distributions without moments. For the standard normal and the classical standard $t$ distributions with 10 degrees of freedom, the Bowley measure is 0 . The Moors measure for these distributions is 1.2331 and 1.27705 , respectively.

In Figure 4, we plot Bowley measure (holding $b=0.5, \gamma=1$ and $\theta=0.1$ fixed) as a function of $c$ for fixed values of $a$ (left) and Bowley measure (holding $a=0.5, \gamma=1$ and $\theta=0.1$ fixed) as a function of $c$ for some values of $b$ (right). In Figure 5, we plot Moors measure (for $b=0.5$, $\gamma=1$ and $\theta=0.1$ ) as a function of $c$ for selected values of $a$ (left) and Moors measure (for $a=0.5, \gamma=1$ and $\theta=0.1$ ) as a function of $c$ for some values of $b$ (right). These plots indicate that these measures can be sensitive to the three shape parameters $a, b$ and $c$. 

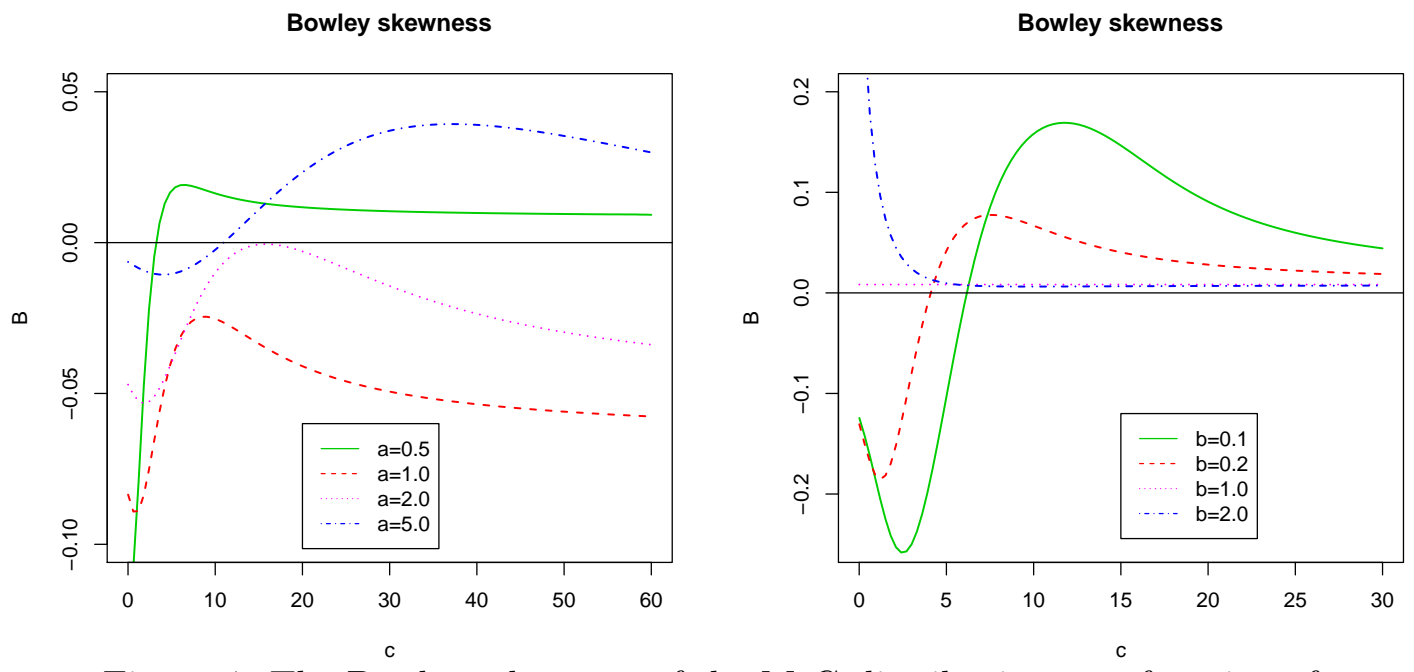

Figure 4: The Bowleys skewness of the McG distribution as a function of $c$.
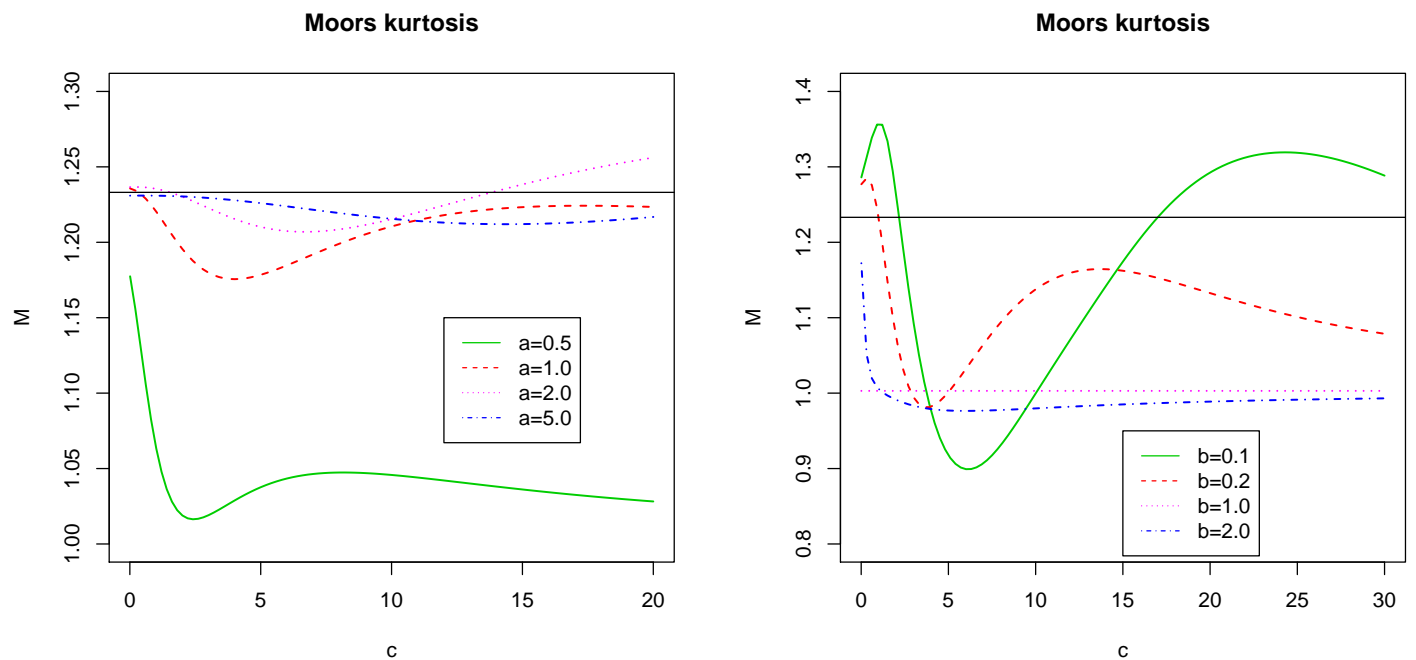

Figure 5: The Moors skewness of the McG distribution as a function of $c$.

\subsection{Entropy}

The entropy of random variable is defined in terms of its probability distribution and can be shown to be a good measure of randomness or uncertainty. The Shannon's entropy of a continuous random variable $Y$ with pdf $f(y)$ is defined by Shannon (1948) as

$$
H_{S h}(f)=-E_{f}[\log f(Y)]=-\int_{0}^{\infty} f(y) \log f(y) d y
$$

Hence, the Shannon entropy for McG distribution can be expressed in the form

$$
H_{S h}(f)=\log \left(\frac{B(a / c, b)}{c \theta}\right)-\theta / \gamma-\gamma E(Y)+\theta / \gamma M_{Y}(\gamma)+(a-1) \zeta(a, b)+(b-1) \zeta(b, a),
$$

where $\zeta(r, s)=\psi(r+s)-\psi(r)$ and $\psi($.$) represents the digamma function. The last two terms in$ (3.7) follows immediately from the first two conditions in Lemma 1 of Zografos and Balakrishnan 
(2009). The Rényi entropy is defined by

$$
H_{\rho}(f)=\frac{1}{1-\rho} \log \left(\int_{-\infty}^{\infty}[f(y)]^{\rho} d y\right)
$$

where $\rho>0$ and $\rho \neq 1$. The Shannon entropy is derived from $\lim _{\rho \rightarrow 1} H_{\rho}(f)$. An explicit expression of Rényi entropy for McG distribution is obtained as

$$
\begin{aligned}
H_{\rho}(f)= & -\log (\theta)+\frac{\rho}{1-\rho} \log \left(\frac{c}{B(a / c, b)}\right)+\frac{1}{1-\rho} \log (B(a \rho-\rho+c j+1, \rho)) \\
& +\frac{1}{1-\rho} \log \left(\sum_{j=0}^{\infty}(-1)^{j}\left(\begin{array}{c}
b \rho-\rho \\
j
\end{array}\right)\right) E\left\{(1-\gamma / \theta \log (1-U))^{\rho-1}\right\},
\end{aligned}
$$

where $U$ has a beta distribution with parameters $a \rho-\rho+c j-1$ and $\rho$.

\section{Estimation}

Let $Y_{1}, \ldots, Y_{n}$ be a random sample of size $n$ from the $M c G(a, b, c, \theta, \gamma)$ distribution and $\Theta=$ $(a, b, c, \theta, \gamma)$ be the unknown parameter vector. The log-likelihood function is given by

$$
\begin{aligned}
l(\boldsymbol{\Theta})= & n \log (c \theta)-n \log (B(a / c, b))+\gamma \sum_{i=1}^{n} y_{i}-\frac{\theta}{\gamma} \sum_{i=1}^{n}\left(e^{\gamma y_{i}}-1\right) \\
& +(a-1) \sum_{i=1}^{n} \log \left(1-t_{i}\right)+(b-1) \sum_{i=1}^{n} \log \left(1-\left(1-t_{i}\right)^{c}\right),
\end{aligned}
$$

where $t_{i}=\exp \left(-\frac{\theta}{\gamma}\left(e^{\gamma y_{i}}-1\right)\right)$. The maximum likelihood estimation (MLE) of $\boldsymbol{\Theta}$ is obtained by solving the nonlinear equations, $U(\boldsymbol{\Theta})=\left(U_{a}(\boldsymbol{\Theta}), U_{b}(\boldsymbol{\Theta}), U_{c}(\boldsymbol{\Theta}), U_{\theta}(\boldsymbol{\Theta}), U_{\gamma}(\boldsymbol{\Theta})\right)^{T}=\mathbf{0}$, where

$$
\begin{aligned}
U_{a}(\boldsymbol{\Theta})= & \frac{\partial l(\boldsymbol{\Theta})}{\partial a}=n / c[\psi(a / c+b)-\psi(a / c)]+\sum_{i=1}^{n} \log \left(1-t_{i}\right), \\
U_{b}(\boldsymbol{\Theta})= & \frac{\partial l(\boldsymbol{\Theta})}{\partial b}=n[\psi(a / c+b)-\psi(b)]+\sum_{i=1}^{n} \log \left(1-\left(1-t_{i}^{c}\right)\right), \\
U_{c}(\boldsymbol{\Theta})= & \frac{\partial l(\boldsymbol{\Theta})}{\partial c}=n / c-n a / c^{2}[\psi(a / c+b)-\psi(a / c)] \\
& -(b-1) \sum_{i=1}^{n} \frac{\left(1-t_{i}^{c}\right) \log \left(1-t_{i}\right)}{1-\left(1-t_{i}\right)^{c}}, \\
U_{\theta}(\boldsymbol{\Theta})= & \frac{\partial l(\boldsymbol{\Theta})}{\partial \theta}=n / \theta-1 / \gamma \sum_{i=1}^{n}\left(e^{\gamma y_{i}}-1\right)+(a-1) / \gamma \sum_{i=1}^{n} \frac{t_{i}\left(e^{\gamma y_{i}}-1\right)}{1-t_{i}} \\
& -c(b-1) / \gamma \sum_{i=1}^{n} \frac{t_{i}\left(1-t_{i}\right)^{c-1}\left(e^{\gamma y_{i}}-1\right)}{1-\left(1-t_{i}\right)^{c}}, \\
U_{\gamma}(\boldsymbol{\Theta})= & \frac{\partial l(\boldsymbol{\Theta})}{\partial \gamma}=\sum_{i=1}^{n} y_{i}+\theta / \gamma^{2} \sum_{i=1}^{n}\left(e^{\gamma y_{i}}-\gamma y_{i} e^{\gamma y_{i}}-1\right)
\end{aligned}
$$




$$
\begin{aligned}
& +\theta(a-1) / \gamma^{2} \sum_{i=1}^{n} \frac{t_{i}\left(\gamma y_{i} e^{\gamma y_{i}}-e^{\gamma y_{i}}+1\right)}{1-t_{i}} \\
& +\theta(b-1) c / \gamma^{2} \sum_{i=1}^{n} \frac{t_{i}\left(1-t_{i}\right)^{c-1}\left(e^{\gamma y_{i}}-\gamma y_{i} e^{\gamma y_{i}}-1\right)}{1-\left(1-t_{i}\right)^{c}} .
\end{aligned}
$$

We need the observed information matrix for interval estimation and hypotheses tests on the model parameters. The $5 \times 5$ Fisher information matrix, $J=J_{n}(\boldsymbol{\Theta})$, is given by

$$
J=-\left[\begin{array}{ccccc}
J_{a a} & J_{a b} & J_{a c} & J_{a \theta} & J_{a \gamma} \\
J_{b a} & J_{b b} & J_{b c} & J_{b \theta} & J_{b \gamma} \\
J_{c a} & J_{c b} & J_{c c} & J_{c \theta} & J_{c \gamma} \\
J_{\theta a} & J_{\theta b} & J_{\theta c} & J_{\theta \theta} & J_{\theta \gamma} \\
J_{\gamma a} & J_{\gamma b} & J_{\gamma c} & J_{\gamma \theta} & J_{\gamma \gamma}
\end{array}\right]
$$

where the expressions for the elements of $J$ are

$$
\begin{aligned}
& J_{a a}=\frac{\partial^{2} l(\boldsymbol{\Theta})}{\partial a^{2}}=\frac{n}{c^{2}}\left[\psi^{\prime}(a / c+b)-\psi^{\prime}(a / c)\right], \\
& J_{a b}=\frac{\partial^{2} l(\boldsymbol{\Theta})}{\partial a \partial b}=\frac{n}{c} \psi^{\prime}(a / c+b), \\
& J_{a c}=\frac{\partial^{2} l(\boldsymbol{\Theta})}{\partial a \partial c}=-\frac{n a}{c^{3}}\left[\psi^{\prime}(a / c+b)-\psi^{\prime}(a / c)\right], \\
& J_{a \theta}=\frac{\partial^{2} l(\boldsymbol{\Theta})}{\partial a \partial \theta}=\frac{1}{\gamma} \sum_{i=1}^{n} \frac{t_{i}\left(e^{\gamma y_{i}}-1\right)}{1-t_{i}} \\
& J_{a \gamma}=\frac{\partial^{2} l(\boldsymbol{\Theta})}{\partial a \partial \gamma}=\frac{\theta}{\gamma^{2}} \sum_{i=1}^{n} \frac{t_{i}\left(\gamma y_{i} e^{\gamma y_{i}}-e^{\gamma y_{i}}+1\right)}{1-t_{i}} \text {, } \\
& J_{b b}=\frac{\partial^{2} l(\boldsymbol{\Theta})}{\partial b^{2}}=n\left[\psi^{\prime}(a / c+b)-\psi^{\prime}(b)\right], \\
& J_{b c}=\frac{\partial^{2} l(\boldsymbol{\Theta})}{\partial b \partial c}=-\frac{n a}{c^{2}} \psi^{\prime}(a / c+b)-\sum_{i=1}^{n} \frac{\left(1-t_{i}\right)^{c} \log \left(1-t_{i}\right)}{1-\left(1-t_{i}\right)^{c}}, \\
& J_{b \theta}=\frac{\partial^{2} l(\boldsymbol{\Theta})}{\partial b \partial \theta}=-\frac{c}{\gamma} \sum_{i=1}^{n} \frac{t_{i}\left(1-t_{i}\right)^{c-1}\left(e^{\gamma y_{i}}-1\right)}{1-\left(1-t_{i}\right)^{c}}, \\
& J_{b \gamma}=\frac{\partial^{2} l(\boldsymbol{\Theta})}{\partial b \partial \gamma}=-\frac{c \theta}{\gamma^{2}} \sum_{i=1}^{n} \frac{t_{i}\left(1-t_{i}\right)^{c-1}\left(\gamma y_{i} e^{\gamma y_{i}}-e^{\gamma y_{i}}+1\right)}{1-\left(1-t_{i}\right)^{c}}, \\
& J_{c c}=\frac{\partial^{2} l(\boldsymbol{\Theta})}{\partial c^{2}}=-\frac{n}{c^{2}}+\frac{2 n a}{c^{3}}[\psi(a / c+b)-\psi(a / c)] \\
& +\frac{n a^{2}}{c^{4}}\left[\psi^{\prime}(a / c+b)-\psi^{\prime}(a / c)\right]-(b-1) \sum_{i=1}^{n} \frac{\left(1-t_{i}\right)^{c}\left(\log \left(1-t_{i}\right)\right)^{2}}{\left[1-\left(1-t_{i}\right)^{c}\right]^{2}}, \\
& J_{c \theta}=\frac{\partial^{2} l(\boldsymbol{\Theta})}{\partial c \partial \theta}=-\frac{b-1}{\gamma} \sum_{i=1}^{n} \frac{t_{i}\left(1-t_{i}\right)^{c-1}\left(e^{\gamma y_{i}}-1\right)\left[c \log \left(1-t_{i}\right)+1-\left(1-t_{i}\right)^{c}\right]}{\left[1-\left(1-t_{i}\right)^{c}\right]^{2}}, \\
& J_{c \gamma}=\frac{\partial^{2} l(\boldsymbol{\Theta})}{\partial c \partial \gamma}=-\frac{\theta(b-1)}{\gamma^{2}} \sum_{i=1}^{n} \frac{t_{i}\left(\gamma y_{i} e^{\gamma y_{i}}-e^{\gamma y_{i}}+1\right)\left[c \log \left(1-t_{i}\right)+1-\left(1-t_{i}\right)^{c}\right]}{\left(1-t_{i}\right)^{1-c}\left[1-\left(1-t_{i}\right)^{c}\right]^{2}},
\end{aligned}
$$




$$
\begin{aligned}
J_{\theta \theta}= & \frac{\partial^{2} l(\boldsymbol{\Theta})}{\partial \theta^{2}}=-\frac{n}{\theta^{2}}-\frac{a-1}{\gamma^{2}} \sum_{i=1}^{n} \frac{t_{i}\left(e^{\gamma y_{i}}-1\right)^{2}}{\left(1-t_{i}\right)^{2}}-\frac{c(b-1)}{\gamma^{2}} \\
& \times \sum_{i=1}^{n} \frac{t_{i}\left(1-t_{i}\right)^{c-2}\left(e^{\gamma y_{i}}-1\right)^{2}\left\{c t_{i}+\left(1-t_{i}\right)^{c}-1\right\}}{\left(1-\left(1-t_{i}\right)^{c}\right)^{2}}, \\
J_{\theta \gamma}= & \frac{\partial^{2} l(\boldsymbol{\Theta})}{\partial \theta \partial \gamma}=\frac{1}{\gamma^{2}} \sum_{i=1}^{n}\left(e^{\gamma y_{i}}-\gamma y_{i} e^{\gamma y_{i}}-1\right)-\frac{c(b-1)}{\gamma^{3}} \sum_{i=1}^{n} \frac{t_{i}\left(e^{\gamma y_{i}}-\gamma y_{i} e^{\gamma y_{i}}-1\right)}{\left(1-t_{i}\right)^{2-c}\left(1-\left(1-t_{i}\right)^{c}\right)^{2}} \\
& \times\left[\left(1-t_{i}\right)^{c}\left(\theta e^{\gamma y_{i}}+t_{i} \gamma-\gamma-\theta\right)+c \theta t_{i}\left(e^{\gamma y_{i}}-1\right)+\gamma\left(1-t_{i}\right)+\theta\left(1-e^{\gamma y_{i}}\right)\right] \\
& +\frac{(a-1)}{\gamma^{3}} \sum_{i=1}^{n} \frac{t_{i}\left(e^{\gamma y_{i}}-\gamma y_{i} e^{\gamma y_{i}}-1\right)\left(\theta e^{\gamma y_{i}}+\gamma t_{i}-\gamma-\theta\right)}{\left(1-t_{i}\right)^{2}}, \\
J_{\gamma \gamma=} & \frac{\partial^{2} l(\boldsymbol{\Theta})}{\partial \gamma^{2}}=\frac{2 \theta}{\gamma^{3}} \sum_{i=1}^{n}\left(\gamma y_{i} e^{\gamma y_{i}}-e^{\gamma y_{i}}-\gamma^{2} y_{i}^{2} e^{\gamma y_{i}} / 2+1\right) \\
& -\frac{c(b-1) \theta^{2}}{\gamma^{4}} \sum_{i=1}^{n} \frac{t_{i}^{2}\left(1-t_{i}\right)^{c-2}\left(\gamma y_{i} e^{\gamma y_{i}}-e^{\gamma y_{i}}+1\right)^{2}\left(2 c\left(1-t_{i}\right)^{c}+\left(1-t_{i}\right)^{c}-c-1\right)}{\left(1-\left(1-t_{i}\right)^{c}\right)^{2}} \\
& +\frac{c(b-1) \theta}{\gamma^{4}} \sum_{i=1}^{n} \frac{t_{i}\left(1-t_{i}\right)^{c-1}}{1-\left(1-t_{i}\right)^{c}}\left[-\gamma^{3} y_{i}^{2} e^{\gamma y_{i}}+2 \gamma^{2} y_{i} e^{\gamma y_{i}}-2 \gamma e^{\gamma y_{i}}+2 \gamma+\theta \gamma^{2} y_{i}^{2} e^{2 \gamma y_{i}}\right. \\
& \left.+\theta\left(e^{\gamma y_{i}}-1\right)^{2}-2 \theta \gamma y_{i} e^{\gamma y_{i}}\left(e^{\gamma y_{i}}-1\right)\right] \\
& -\frac{(a-1) \theta}{\gamma^{4}} \sum_{i=1}^{n} \frac{t_{i}}{1-t_{i}}\left[-\gamma^{3} y_{i}^{2} e^{\gamma y_{i}}+2 \gamma^{2} y_{i} e^{\gamma y_{i}}-2 \gamma\left(e^{\gamma y_{i}}-1\right)+\theta\left(\gamma y_{i} e^{\gamma y_{i}}-e^{\gamma y_{i}}+1\right)^{2}\right] \\
& -\frac{(a-1) \theta^{2}}{\gamma^{4}} \sum_{i=1}^{n} \frac{t_{i}^{2}\left(\gamma y_{i} e^{\gamma y_{i}}-e^{\gamma y_{i}}+1\right)^{2}}{\left(1-t_{i}\right)^{2}} .
\end{aligned}
$$

Under conditions that are fulfilled for parameters in the interior of the parameter space but not on the boundary, asymptotically

$$
\sqrt{n}(\hat{\boldsymbol{\Theta}}-\boldsymbol{\Theta}) \sim N_{5}\left(0, I(\boldsymbol{\Theta})^{-1}\right)
$$

where $I(\boldsymbol{\Theta})$ is the expected information matrix. This asymptotic behavior is valid if $I(\Theta)$ replaced by $J_{n}(\hat{\boldsymbol{\Theta}})$, i.e., the observed information matrix evaluated at $\hat{\boldsymbol{\Theta}}$ Cox and Hinkley (1979).

\section{Application of McG to two real data sets}

In this section, two real data sets are considered to illustrate that the McG model can be a good lifetime distribution comparing with main three submodels; BG, KumG and McE distributions. In both examples, we obtain the MLE and their corresponding standard errors (in parentheses) of the model parameters. The model selection is carried out using minus of log-likelihood function $(-\log (L))$, Kolmogorov-Smirnov (K-S) statistic with its p-value, Akaike information 
criterion (AIC), Akaike information criterion corrected (AICC), Bayesian information criterion (BIC) and likelihood ratio test (LRT) with its p-value. Furthermore, we plot the histogram for each data set and the estimated pdf of the four models. Moreover, the plots of empirical cdf of the data sets and estimated cdf of four models are displayed.

Example 5.1. The data set have been obtained from Aarset (198\%) and represents the lifetimes of 50 devices. Also, it is analyzed by El-Gohary et al. (2013) and Jafari et al. (2014). The results which are given in Table 1 indicate that the McE model is not suitable for this data set based on K-S statistic. The McG model has the lowest $-\log (L), A I C$ and AICC values among all fitted models, but the BG model has the lowest BIC value among all other fitted models. A comparison of the proposed distribution with some of its submodels using p-value of LRT shows that the McG model yields a better fit than the other three distributions to this real data set. It is also clear from Figure 6 that the $M c G$ distribution provides a better fit and therefore be one of the best models for this data set.

Example 5.2. The data set represents the strengths of $1.5 \mathrm{~cm}$ glass fibers, measured at the National Physical Laboratory, England. Unfortunately, the units of measurement are not given in the paper. It is obtained from Smith and Naylon (198\%) and also analyzed by Barreto-Souza et al. (2010). The K-S statistic indicates that all fitted models are good candidates for this data set, but based on all other criteria: - $\log (L)$, AIC, AICC, BIC and p-value of LRT, we can infer that the best model is the McG distribution. Similar results are concluded from Figure 7 . 
Table 1: MLEs of the model parameters for the data of lifetimes of 50 devices, the corresponding SEs and the K-S, AIC, AICC, BIC and LRT statistics.

\begin{tabular}{|l|c|c|c|c|}
\hline & \multicolumn{4}{|c|}{ Distribution } \\
\hline$\hat{a}$ & BG & KumG & McE & McG \\
(s.e.) & 0.2158 & 0.2374 & 0.8643 & 0.2619 \\
$\hat{b}$ & $(0.0392)$ & $(0.0871)$ & $(0.4621)$ & $(0.0656)$ \\
(s.e.) & 0.2467 & 0.6063 & 0.0706 & 0.0752 \\
\hline$\hat{c}$ & $(0.0448)$ & $(0.3780)$ & $(0.0927)$ & $(0.1029)$ \\
(s.e.) & - & 0.2374 & 2.7127 & 3.7652 \\
$\hat{\theta}$ & - & $(0.0871)$ & $(3.7438)$ & $(0.9946)$ \\
(s.e.) & 0.0003 & 0.0003 & 0.2826 & 0.0012 \\
$\hat{\gamma}$ & $(0.0001)$ & $(0.0001)$ & $(0.3727)$ & $(0.0001)$ \\
(s.e.) & 0.0882 & 0.0766 & - & 0.0875 \\
\hline \hline$-\log (L)$ & $(0.0030)$ & $(0.0282)$ & - & $(0.0001)$ \\
K-S & 220.6714 & 221.9666 & 237.8158 & 219.0041 \\
p-value (K-S) & 0.1322 & 0.1367 & 0.1916 & 0.1216 \\
AIC & 0.3456 & 0.3072 & 0.0507 & 0.4509 \\
AICC & 449.3437 & 451.9331 & 483.6316 & 448.0081 \\
BIC & 450.2326 & 452.8220 & 484.5205 & 449.3718 \\
LRT & 456.9918 & 459.5812 & 491.2797 & 457.5682 \\
p-value (LRT) & 3.3356 & 5.9249 & 37.6233 & - \\
\hline
\end{tabular}

Table 2: MLEs of the model parameters for the strengths of $1.5 \mathrm{~cm}$ glass fibers data, the corresponding SEs and the K-S, AIC, AICC, BIC and LRT statistics.

\begin{tabular}{|l|c|c|c|c|}
\hline & \multicolumn{4}{|c|}{ Distribution } \\
\hline & BG & KumG & McE & McG \\
\hline$\hat{a}$ & 1.6907 & 1.8946 & 9.3276 & 0.7940 \\
(s.e.) & $(0.8664)$ & $(1.2350)$ & $(2.6891)$ & $(0.2355)$ \\
\hline$\hat{b}$ & 27.7434 & 4.2814 & 93.4655 & 0.1248 \\
(s.e.) & $(87.8726)$ & $(15.7612)$ & $(109.2042)$ & $(0.1786)$ \\
\hline$\hat{c}$ & - & 1.8946 & 22.6124 & 192.1704 \\
(s.e.) & - & $(1.2350)$ & $(14.0674)$ & $(307.3698)$ \\
\hline$\hat{\theta}$ & 0.0020 & 0.0309 & 0.9227 & 0.0009 \\
(s.e.) & $(0.0632)$ & $(0.0509)$ & $(0.3443)$ & $(0.0001)$ \\
\hline$\hat{\gamma}$ & 2.7156 & 2.3019 & - & 5.2013 \\
(s.e.) & $(0.8448)$ & $(1.6517)$ & - & $(0.4018)$ \\
\hline \hline$-\log (L)$ & 14.2158 & 14.0305 & 15.5995 & 11.4208 \\
K-S & 0.1324 & 0.1313 & 0.1466 & 0.1159 \\
p-value (K-S) & 0.2186 & 0.2271 & 0.1335 & 0.3651 \\
AIC & 36.4317 & 36.0610 & 37.2569 & 32.8417 \\
AICC & 37.1213 & 36.7506 & 37.9466 & 33.8943 \\
BIC & 45.0042 & 44.6335 & 45.8295 & 43.5573 \\
LRT & 5.5900 & 5.2193 & 8.3572 & - \\
p-value (LRT) & 0.0181 & 0.0223 & 0.0038 & - \\
\hline
\end{tabular}



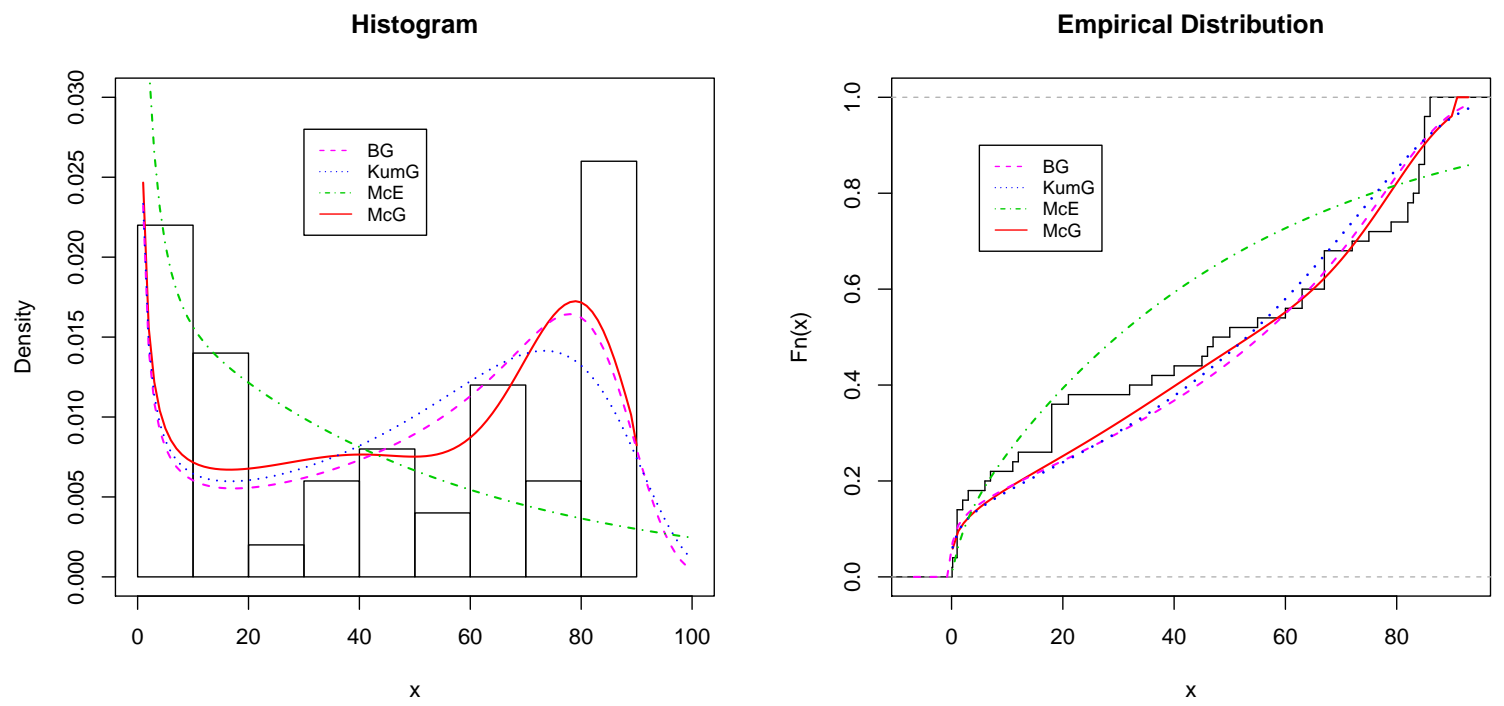

Figure 6: Plots of the estimated pdfs and cdfs BG, KumG, McE and McG models using data of lifetimes of 50 devices.
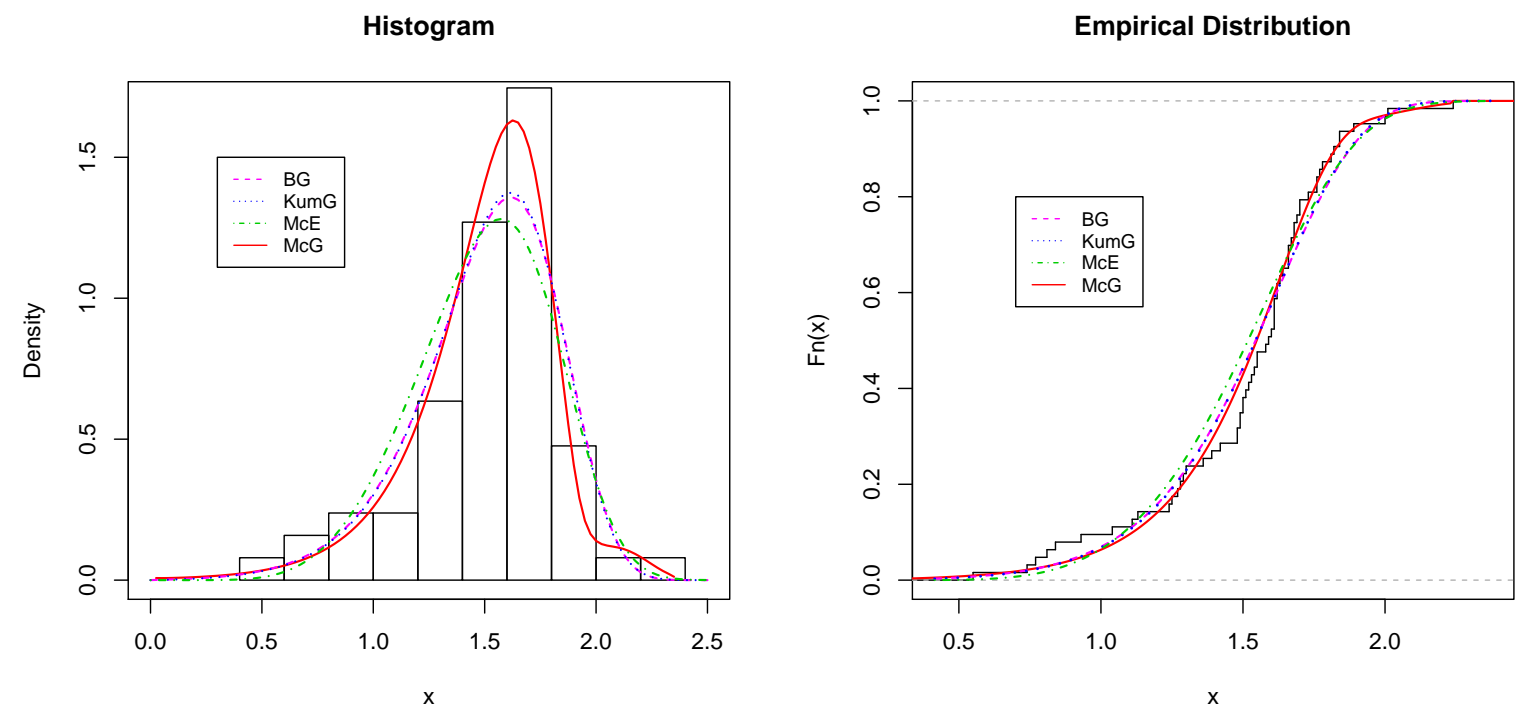

Figure 7: Plots of the estimated pdfs and cdfs BG, KumG, McE and McG models using the strengths of $1.5 \mathrm{~cm}$ glass fibers data. 


\section{References}

Aarset, M. V. (1987). How to identify a bathtub hazard rate. IEEE Transactions on Reliability, R-36(1):106-108.

Alexander, C., Cordeiro, G. M., Ortega, E. M. M., and Sarabia, J. M. (2012). Generalized beta-generated distributions. Computational Statistics and Data Analysis, 56(6):1880-1897.

Barreto-Souza, W., Santos, A. H. S., and Cordeiro, G. M. (2010). The beta generalized exponential distribution. Journal of Statistical Computation and Simulation, 80(2):159-172.

Bemmaor, A. C. and Glady, N. (2012). Modeling purchasing behavior with sudden death: A flexible customer lifetime model. Management Science, 58(5):1012-1021.

Brown, K. and Forbes, W. (1974). A mathematical model of aging processes. Journal of Gerontology, 29(1):46-51.

Cordeiro, G. M., Cintra, R. J., Rêgo, L. C., and Ortega, E. M. (2012a). The McDonald normal distribution. Pakistan Journal of Statistics and Operation Research, 8(3):301-329.

Cordeiro, G. M., Hashimoto, E. M., Ortega, E. M., and Pascoa, M. A. (2012b). The McDonald extended distribution: properties and applications. AStA Advances in Statistical Analysis, 96(3):409-433.

Cordeiro, G. M., Hashimoto, E. M., and Ortega, E. M. M. (2014). The McDonald Weibull model. Statistics, 48(2):256-278.

Cordeiro, G. M. and Lemonte, A. J. (2012). The McDonald inverted beta distribution. Journal of the Franklin Institute, 349(3):1174-1197.

Cordeiro, G. M. and Lemonte, A. J. (2014). The McDonald arcsine distribution: a new model to proportional data. Statistics, 48(1):182-199.

Cox, D. R. and Hinkley, D. V. (1979). Theoretical Statistics. Chapman and Hall, London.

Economos, A. C. (1982). Rate of aging, rate of dying and the mechanism of mortality. Archives of Gerontology and Geriatrics, 1(1):46-51.

El-Gohary, A., Alshamrani, A., and Al-Otaibi, A. N. (2013). The generalized Gompertz distribution. Applied Mathematical Modelling, 37(1-2):13-24. 
Gradshteyn, I. S. and Ryzhik, I. M. (2007). Table of Integrals, Series, and Products, Edited by Alan Jeffrey and Daniel Zwillinger. Academic Press, New York, 7th edition.

Gupta, R. D. and Kundu, D. (1999). Generalized exponential distributions. Australian \& New Zealand Journal of Statistics, 41(2):173-188.

Jafari, A. A., Tahmasebi, S., and Alizadeh, M. (2014). The beta-Gompertz distribution. Revista Colombiana de Estadística, 37(1):141-158.

Johnson, N. L., Kotz, S., and Balakrishnan, N. (1995). Continuous Univariate Distributions, volume 2. John Wiley \& Sons, New York, second edition.

Kenney, J. F. and Keeping, E. (1962). Mathematics of Statistics. D. Van Nostrand Company.

Manoj, C., Wijekoon, P., and Yapa, R. D. (2013). The McDonald generalized beta-binomial distribution: A new binomial mixture distribution and simulation based comparison with its nested distributions in handling overdispersion. International Journal of Statistics and Probability, 2(2):p24.

Marciano, F. W. P., Nascimento, A. D. C., Santos-Neto, M., and Cordeiro, G. M. (2012). The Mc- $\Gamma$ distribution and its statistical properties: An application to reliability data. International Journal of Statistics and Probability, 1(1):p53.

McDonald, J. B. (1984). Some generalized functions for the size distribution of income. Econometrica, 52:647-663.

Moors, J. J. A. (1988). A quantile alternative for kurtosis. Journal of the Royal Statistical Society. Series D (The Statistician), 37(1):25-32.

Mudholkar, G. S. and Natarajan, R. (2002). The inverse gaussian models: analogues of symmetry, skewness and kurtosis. Annals of the Institute of Statistical Mathematics, 54(1):138-154.

Mudholkar, G. S. and Wang, H. (2007). IG-symmetry and R-symmetry: interrelations and applications to the inverse gaussian theory. Journal of Statistical Planning and Inference, 137(11):3655-3671.

Nadarajah, S., Cordeiro, G. M., and Ortega, E. M. (2012). General results for the kumaraswamy-g distribution. Journal of Statistical Computation and Simulation, 82(7):951979. 
Nadarajah, S. and Kotz, S. (2006). The beta exponential distribution. Reliability Engineering Es System Safety, 91(6):689-697.

Oliveira, J., Santos, J., Xavier, C., Trindade, D., and Cordeiro, G. M. (2013). The McDonald half-logistic distribution: Theory and practice. Communications in Statistics-Theory and Methods, 10.1080/03610926.2013.873131.

Oluyede, B. O. and Rajasooriya, S. (2013). The Mc-Dagum distribution and its statistical properties with applications. Asian Journal of Mathematics and Applications, 2013:ama0085.

Shannon, C. (1948). A mathematical theory of communication. Bell System Technical Journal, $27: 379-432$.

Smith, R. L. and Naylor, J. C. (1987). A comparison of maximum likelihood and Bayesian estimators for the three-parameter Weibull distribution. Applied Statistics, 36(3):358-369.

Tahir, M., Mansoor, M., Zubair, M., and Hamedani, G. (2014). McDonald log-logistic distribution with an application to breast cancer data. Journal of Statistical Theory and Applications, 13(1):65-82.

Zografos, K. and Balakrishnan, N. (2009). On families of beta-and generalized gammagenerated distributions and associated inference. Statistical Methodology, 6(4):344-362. 